\title{
Influence of Pre-Impregnation Treatment on Bamboo Reinforced Epoxy/UPE Resin Composites
}

\author{
Pradeep Kr. Kushwaha ${ }^{1}$, Rakesh Kumar ${ }^{2}$ \\ ${ }^{1}$ Indian Plywood Industries Research and Training Institute, Mohali, India; ${ }^{2}$ Indian Institute of Technology, New Delhi, India. \\ Email: pradeepkk@ipirti.gov.in
}

Received July $25^{\text {th }}, 2012$; revised August $24^{\text {th }}, 2012$; accepted September $7^{\text {th }}, 2012$

\begin{abstract}
Bamboo based biocomposites were made with woven bamboo mats and epoxy/unsaturated polyester (UPE) resins. The effects of pre-impregnation on the mechanical and water resistant properties of composites were studied. An alkaline treatment was used to enhance the fiber wetting so as to achieve complete pre-impregnation with epoxy and unsaturated polyester resins. A combined treatment of alkali and dilute epoxy/polyester gives the best result. Bending strength, modulus of elasticity, tensile strength, tensile modulus, impact strength, water absorption were evaluated and compared for all composites. Scanning electron microscopy (SEM) studies of the composites tensile fracture surface also indicated that there is an improved adhesion between fiber and matrix. The mechanical as well as water resistant properties of the biocomposites improved after pre-impregnation treatment.
\end{abstract}

Keywords: Biocomposites; Pre-Impregnation; Epoxy Resin; Polyester Resin; Mechanical Properties

\section{Introduction}

Many papers are published about the pre-impregnated natural fiber reinforced composites [1-4]. A combined treatment of alkali and dilute epoxy gives the best result. The epoxy has been diluted in acetone, and tests show that this has a significant effect on the mechanical properties of the composite [1]. The adhesion between industrial hemp fibers and UPE matrix was increased by treatment of hemp fiber surface with UPE (matrix). The surface treatment of hemp mats also resulted in higher mechanical and thermal properties [2]. Henequen fiber surface preimpregnation with dilute polyethylene dilute solution results in composite with increased tensile strength and interfacial shear strength $[3,4]$.

In this study, resin pre-impregnation of bamboo matting was carried out by dilute epoxy and polyester resins. Pre-treated bamboo reinforced epoxy and polyester composites were prepared and the properties were measured. The effect of treatment on tensile, flexural and water absorption properties were examined.

\section{Experimental}

Orthogonal bamboo strip mats were acquired from the local market. The cross-section of the strip used in the mats is $4.25 \mathrm{~mm} \times 0.5 \mathrm{~mm}$. Bamboo mats cut to size $300 \times 200 \mathrm{~mm}^{2}$ were cleaned with washing powder and washed in running water thoroughly to remove dust and other deposits from the surface. They were left in the open to dry out for $4 \mathrm{~h}$. Epoxy resin (CY-230) and hardener (HY-951) were purchased from CIBA-GEIGY. Epoxy resin was heated in oven for $10 \mathrm{~min}$ to remove the moisture and air bubbles. After cooling the resin to room temperature, hardener, $10 \%$ by weight, was added, stirred to mix thoroughly and subsequently applied on the bamboo mats. Piled one over another to seven layers. Placed in between two Perspex sheets, $300 \times 200 \mathrm{~mm}^{2}$, and pressed in the hydraulic press under a pressure of 170 $\mathrm{KN}$ for $24 \mathrm{~h}$. Then the composites were cured at $80^{\circ} \mathrm{C}$ for $4 \mathrm{~h}$ in oven.

The bamboo mats were alkali treated before preimpregnation. The fiber mats were completely immersed in five percent solution of sodium hydroxide solution for half an hour at room temperature. Then the fiber mats were washed with tap water until adhered alkali was removed from their surface. The fiber mats were then neutralized in distilled water with $2 \%$ glacial acetic acid. This solution was then drained and the fibers were washed with distilled water and kept for drying in the open. After room drying, they were oven dried at $80^{\circ} \mathrm{C}$ for $8 \mathrm{~h}$.

For the pre-impregnation with dilute epoxy, the alkali treated fibres were immersed in $2 \%$ solution of epoxy which was dissolved in acetone for $2 \mathrm{~h}$ at room temperature. After removal, the fibers were dried and cured in an oven at $80^{\circ} \mathrm{C}$ for $8 \mathrm{~h}$. These surface-treated fiber mats 
were then used for fabricating composites with epoxy.

Five percent solution of UPE and MEKP (99:1) was made in $95 \%$ acetone. The alkali treated fibers were soaked in the UPE-MEKP solution for $30 \mathrm{~min}$. The excess solution was drained off from their surface and they were dried overnight under the hood. The treated mats were oven dried at $80^{\circ} \mathrm{C}$ for $8 \mathrm{~h}$. These surface-treated fiber mats were then used for fabricating composites with polyester. A number of composites were fabricated as tabulated in Table 1.

The tensile test was performed according to the ASTM D638. Dog bone shaped specimens of the composites were cut. At least five replicate specimens were tested for each formulation. Each specimen was tested to failure under tension at a cross-head speed of $2 \mathrm{~mm} / \mathrm{min}$ on INSTRON 3369. Three-point bending test was performed according to ASTM D790. Specimens of dimension $96 \times$ $13 \mathrm{~mm}^{2}$ were prepared. Support separation was $70 \mathrm{~mm}$ and test speed was $5 \mathrm{~mm} / \mathrm{min}$ on ZWICK Z010. Notched Izod impact test samples with dimensions of $63.5 \times 12.5$ $\mathrm{mm}^{2}$ by the thickness were cut from the laminate composites. The testing was conducted according to ASTM D256 on a Zwick model 5101 with a pendulum weight of $25 \mathrm{~J}$. Five samples were tested and the average value was taken as the Izod impact strength. The Izod impact strength was calculated using the formula given below:

Impact strength $\left(\mathrm{kJ} / \mathrm{m}^{2}\right)=[$ Impact energy $(\mathrm{J}) /$ Cross sectional area] $\times 10^{3}$.

Tensile fracture surface of the composite samples were coated with silver and then analyzed using CARL ZEISS EVO 50 scanning electron microscope.

Water absorption studies were performed following the ASTM D570-98 method at room temperature $\left(25^{\circ} \mathrm{C}\right)$. The samples were taken out periodically and weighed immediately, after wiping out the water on the surface of the sample, to find out the content of water absorbed. The amount of water absorbed in the composites was calculated by the weight difference between the samples exposed to water and the dried samples.

\section{Results and Discussions}

The alkali treatment removes the hemicellulose, lignin and pectins. The impregnation with dilute epoxy presents an optimum improvement of the longitudinal properties. In this case, the low-viscous resin can already fill the micro-pores and flow in between the elementary fibers, which is beneficial for the impregnation afterwards. In fact, this treatment is the combined effect of three solutions, namely alkali, epoxy and acetone, in which the epoxy has been diluted.

As shown in Figure 1, the tensile strength of treated bamboo epoxy composite is $40 \%$ higher, and the elastic modulus is $35 \%$ higher as compared with that of un-
Table 1. Nomenclature of water modified composites.

\begin{tabular}{ll}
\hline Material & \multicolumn{1}{c}{ Specification } \\
\hline BE & Untreated bamboo epoxy composite \\
BPIE & Epoxy pre-impregnated bamboo epoxy composite \\
BP & Untreated bamboo polyester composite \\
BPIP & Polyester pre-impregnated bamboo polyester composite \\
\hline
\end{tabular}

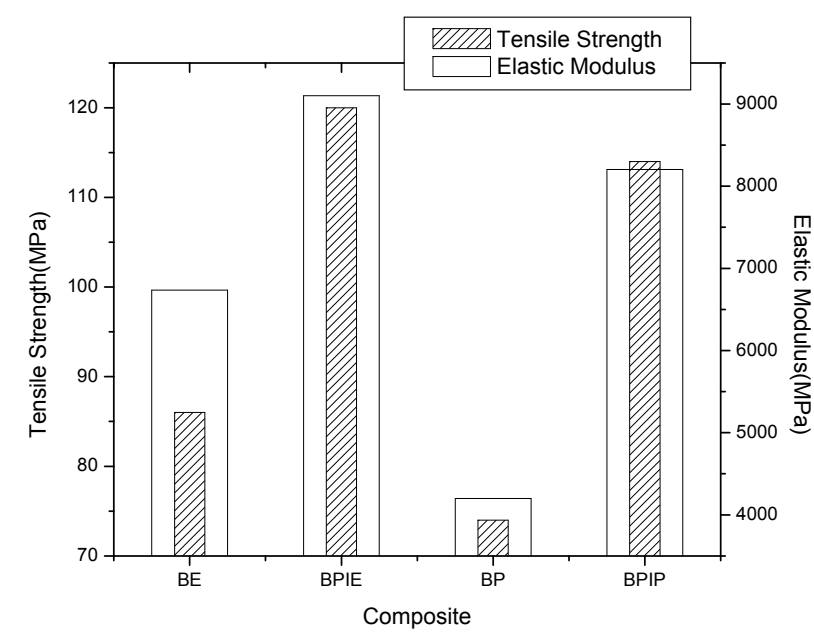

Figure 1. Tensile properties of water treated bamboo composites.

treated bamboo-epoxy composite. The treated bamboo polyester composite has an increment of $54 \%$ in tensile strength, and $95 \%$ in elastic modulus compared with that of untreated bamboo based polyester composite. According to Weyenberg et al. [2], the increase in tensile strength and modulus of the treated hemp fibers-based composites may be an outcome of the improved adhesion between the fiber and the matrix. This improved adhesion might have enhanced the interfacial bonding and thus made it easier for the stress to be effectively transferred from the matrix to the fiber. The adhered matrix over the fiber can be observed from the Figures 2(a) and (b).

As shown in Figure 3, the flexural strength of treated bamboo epoxy composite is $39 \%$ higher, and the flexural modulus show a decrement from $11.9 \mathrm{GPa}$ to $9.8 \mathrm{GPa}$ compared with that of untreated bamboo epoxy composite. The treated bamboo polyester composite has an increment of $20 \%$ in flexural strength, and $116 \%$ in flexural modulus compared with that of untreated bamboo based polyester composite.

Figure 4 shows the water absorption behavior of pre-impregnated bamboo epoxy and polyester composites. The water absorption for all the composites increased up to 5th or 7th week and then leveled-off. In both the procedure of water absorption test, composites reinforced with chemically treated fibers absorb less water 


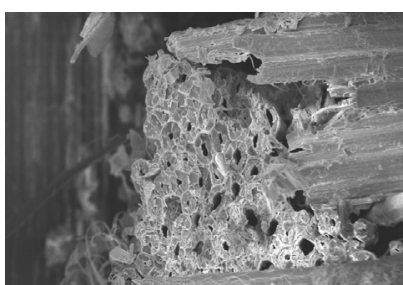

(a)

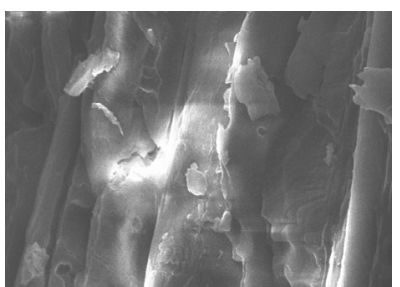

(b)
Figure 2. SEM micrograph of tensile fracture surfaces of (a) BPIE \& (b) BPIP.

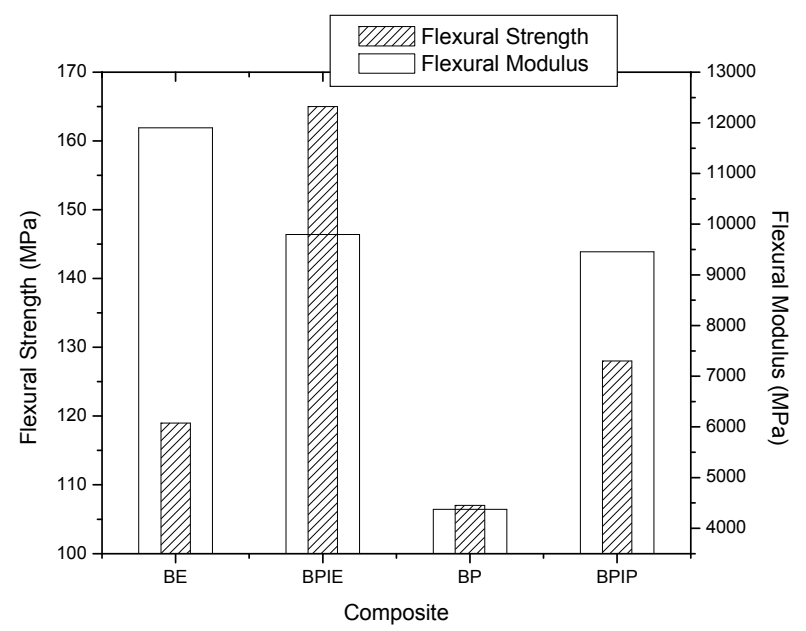

Figure 3. Flexural properties of water treated bamboo composites.

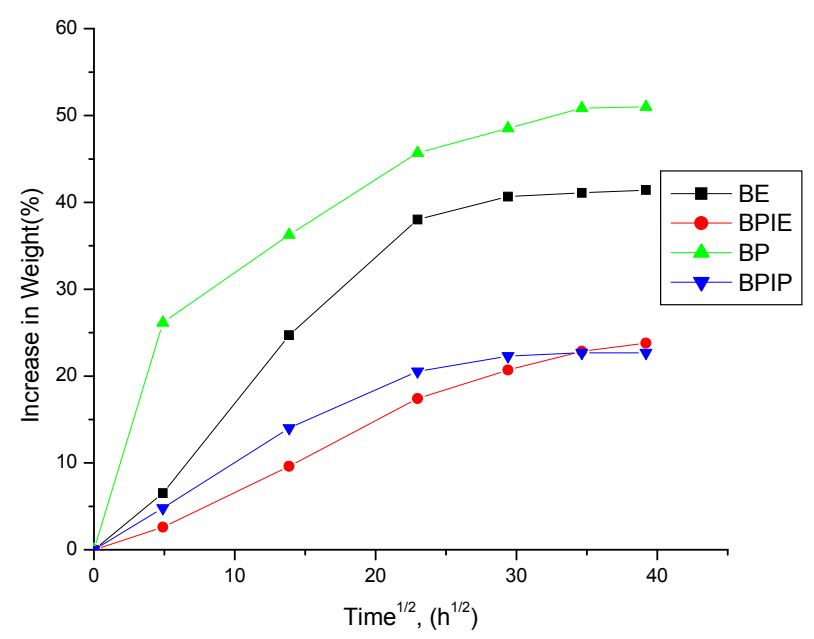

Figure 4. Water absorption curve for water treated bamboo composites.

than the untreated fibers. For the long term water immersion test, percent increase in water absorption for untreated bamboo polyester and epoxy composites are
52.26 and 41.43 respectively. The water absorption by the treated composites has decreased. Water absorption at saturation was $23.80 \%$ and $22.68 \%$ for the epoxy and polyester based composites respectively. This is due to improved adhesion between the fibers and matrix. In this case, the low-viscous resin can already fill the micropores and flow in between the elementary fibers, which is beneficial for the impregnation afterwards.

As shown in Figure 4, where percentage moisture absorption increases steadily with $\mathrm{t}^{1 / 2}$ in the initial stage and then tend to level off following the saturation point, indicating a Fickian mode of diffusion.

\section{Conclusion}

Preimpregnation has improved the mechanical and water resistant properties of the both epoxy and polyester based composites. The tensile strength of preimpregnated bamboo epoxy composite is increased by $40 \%$ and the elastic modulus by $35 \%$. The treated bamboo polyester composite has an increment of 54\% in tensile strength and $95 \%$ in elastic modulus. The treated bamboo polyester composite has an increment of $20 \%$ in flexural strength, and $116 \%$ in flexural modulus. The percentage improvement was more for treated bamboo polyester composite. Water absorption at saturation was $23.80 \%$ and $22.68 \%$ for the epoxy and polyester based composites respectively.

\section{REFERENCES}

[1] G. Mehta, L. T. Drzal, A. K. Mohanty and M. Misra, "Effect of Fiber Surface Treatment on the Properties of Biocomposites from Nonwoven Industrial Hemp Fiber Mats and Unsaturated Polyester Resin," Journal of Applied Polymer Science, Vol. 99, No. 3, 2006, pp. 10551068. doi:10.1002/app.22620

[2] I. Van de Weyenberg, J. Ivens, A. De Coster, B. Kino, B. Baetens and I. Verpoest, "Influence of Processing and Chemical Treatment of Flax Fibres on Their Composites Composites," Science and Technology, Vol. 63, No. 9, 2003, pp. 1241-1246. doi:10.1016/S0266-3538(03)00093-9

[3] A. Valadez-Gonzaleza, J. M. Cervantes-Uca, R. Olayob and P. J. Herrera-Francoa, "Effect of Fiber Surface Treatment on the Fiber-Matrix Bond Strength of Natural Fiber Reinforced Composites," Composites: Part B, Vol. 30, No. 3, 1999, pp. 309-320. doi:10.1016/S1359-8368(98)00054-7

[4] P. J. Herrera-Franco and A. Valadez-González, "A Study of the Mechanical Properties of Short Natural-Fiber Reinforced Composites," Composites: Part B, Vol. 36, No. 8, 2005, pp. 597-608.

doi:10.1016/j.compositesb.2005.04.001 